\title{
Simple tests for septic bursitis: comparative study
}

\author{
I M Stell, W R Gransden
}

Differentiating septic from non-septic bursitis of the olecranon and prepatellar bursae is a common and important problem. Though septic cases may be identified clinically, laboratory tests have also been used. ${ }^{12}$ However these tests are either not widely available or inadequately sensitive. We assessed two new tests that can be performed by most clinical laboratories: estimation of cell counts in a sample of EDTA anticoagulated bursal fluid and inoculation of the fluid into liquid media.

\section{Subjects, methods, and results}

A total of 36 patients (32 men), 28 with olecranon and eight with prepatellar bursitis, were recruited from 54 consecutive patients with these conditions attending an accident and emergency department between May 1996 and March 1997 (age range 21-88 (median 36.5) years). Delay to presentation, severity of pain (visual analogue scale), fever, and degree of bursal erythema, warmth, and tenderness were recorded. The bursa was aspirated and the appearance of the fluid noted. The aspirate was divided into three aliquots, one for absolute and differential cell counts performed on an automated cell counter (Coulter), one for direct culture on solid media, and one for inoculation into liquid media (blood culture bottles, vital, bio Merieux SA). Treatment was in accordance with current practice. The definitive diagnosis of septic or non-septic bursitis was determined for each patient by an independent panel of senior clinicians, not otherwise involved in the study, on the basis of all clinical, laboratory, treatment, and follow up data to the point of final discharge (table). The four patients who did not attend follow up were contacted by telephone.

Patients with septic bursitis usually presented earlier and had more pain, erythema, warmth and tenderness, and some had mild fever (maximum recorded $37.5^{\circ} \mathrm{C}$ ). Clinical features alone could not identify all the septic cases, nor were the volume and appearance of the aspirate helpful.

Positive results were obtained on culture in liquid media in all 17 cases of septic bursitis and on direct culture in 10 cases $(\mathrm{P}<0.05$, McNemar's test with continuity correction). This gives a sensitivity of $100 \%$ (95\% confidence interval $92 \%$ to $108 \%$ ), specificity of $89 \%(74 \%$ to $104 \%)$, and predictive value of a positive test of $89 \%$ (74\% to $104 \%)$. Median white cell counts in septic cases were higher than in non-septic cases $\left(\mathrm{P}<0.001\right.$, Mann-Whitney U test). Counts $>2 \times 10^{9} / 1$ had a sensitivity of $94 \%$ ( $81 \%$ to $107 \%)$, specificity of $79 \%(60 \%$ to $98 \%)$ and positive predictive value of $80 \%$ $(62 \%$ to $98 \%)$ in the detection of septic cases.

Staphylococcus aureus was isolated in 15 of the 17 septic cases, $\beta$ haemolytic streptococcus group $G$ in one, and Staphylococcus epidermidis in one.

\section{Comment}

This greater sensitivity of liquid media inoculation than of direct culture methods in detecting sepsis has also been established for acute monoarthritis. ${ }^{3}$ The main reasons are likely to be the larger inoculum of fluid used in liquid media methods, lysis of leucocytes with release of phagocytosed bacteria, and dilution of inhibitory factors by the medium.

Synovial fluid cell counts have been widely used in diagnosing joint conditions, ${ }^{4}$ mainly by manual microscopy as high viscosity makes samples unsuitable for automated cell counters. These are designed to count and differentiate cells in whole blood, but the principles are applicable to other body fluids. We established the validity of automated cell counters for this fluid in a prior study (unpublished data).

It is standard practice to aspirate inflamed bursae. ${ }^{5}$ With these two investigations, guidance on diagnosis can be obtained rapidly from the cell count, and treatment can be given on the same day. If doubt remains then antibiotics should be given while awaiting culture results. We acknowledge the help of Professor R Grahame, Mr J D Spencer, and the staff of the accident and emergency department of Guy's Hospital and of the haematology and microbiology departments of Guy's and St Thomas's NHS Trust.

Contributors: IMS inititated the study, which was then developed with WRG. IMS organised data collection, performed telephone follow up, and drafted the paper. WRG supervised microbiological analyses and revised the paper. IMS will act as guarantor for the paper.

Funding: None.

Conflict of interest: None.

1 Zimmerman B, Mikolich DJ, Ho G. Septic bursitis. Semin Arth Rheum $1995 ; 24: 391-410$

2 Ho G, Tice AD. Comparison of nonseptic and septic bursitis, further observations on the treatment of septic bursitis. Arch Intern Med 1979:139:1269-73.

3 Von Essen R, Hölttä A. Improved method of isolating bacteria from joint fluids by the use of blood culture bottles. Ann Rheum Dis 1986;45:454-7.

4 Freemont AJ, Denton J, Chuck A, Holt PJL, Davies M. Diagnostic value of synovial fluid microscopy: a reassessment and rationalisation. Ann Rheum Dis 1991;50:101-7.

5 Raddatz DA, Hoffman GS, Franck WA. Septic bursitis, presentation, treatment and prognosis. J Rheumatol 1987;14:1160-3. (Accepted 4 February 1998)

Discrimination of septic and non-septic bursitis by clinical features and laboratory tests

\begin{tabular}{lcc} 
& Septic bursitis ( $\mathbf{n}=\mathbf{1 7})$ & Non-septic bursitis ( $\mathbf{n}=\mathbf{1 9})$ \\
\hline Median (range) onset to presentation (days) & $1(1-7)$ & $2(0-29)$ \\
\hline Median (range) pain (visual analogue score) & $3.6(0.8-7.8)$ & $0.8(0.2-7.0)$ \\
\hline No (\%) with fever & $7(41)$ & 0 \\
\hline No (\%) with erythema & $14(82)$ & $5(26)$ \\
\hline
\end{tabular}

Skin temperature:

\begin{tabular}{lcc}
\hline Cool & 0 & 7 \\
\hline Warm & 12 & 11 \\
\hline Hot & 5 & 1 \\
\hline Tenderness: & 1 & 8 \\
\hline None & 8 & 9 \\
\hline Mild & 4 & 2 \\
\hline Moderate & 4 & 0 \\
\hline Severe & & \\
\hline Appearance of aspirate: & 2 & 3 \\
\hline Clear & 4 & 4 \\
\hline Hazy & 9 & 5 \\
\hline Serosanguinous & 2 & 7 \\
\hline Bloody & $5.2(1.2-34.6) \dagger$ & $1.3(0.1-6.1) \dagger$ \\
\hline Median (range) bursal fluid white cell count $\left(\times 10^{9} /\right)$ & $79(17-99)$ & $36(13-71)$ \\
\hline$\%$ (range) neutrophils & $10(63)^{*}$ & 0 \\
\hline No (\%) positive on direct culture & $17(100)^{\star}$ & $2(11)$ \\
\hline No (\%) positive on culture in liquid media & & \\
\hline
\end{tabular}

College Hospital,

London SE5 9RS
Guy's Hospital, London SE1 9RT I M Stell, lecturer, accident and emergency department W R Gransden, senior lecturer, department of clinical microbiology

Correspondence to: Dr I M Stell, Accident and Emergency Department, King's Healthcare NHS

Trust, King's

BMJ 1998;316:1877 